\title{
Empirical Analysis of Rotation Invariance in Moment Coefficients
}

\author{
Sukhjeet K. Ranade \\ Department of Computer Science \\ Punjabi University Patiala
}

\author{
Supriya Anand \\ Department of Computer Science \\ Punjabi University Patiala
}

\begin{abstract}
Moments can be viewed as powerful image descriptors that capture global characteristics of an image. The magnitude of the moment coefficients is said to be invariant under geometrical transformations like rotation which makes them suitable for most of the recognition applications. But in practice, the invariance of moment coefficients is compromised due to the errors in computation. This paper presents an empirical study of some popularly used moment functions to find out the robust coefficients under rotation. The selected robust coefficients are used in face recognition under in-plane rotation. Experimental results demonstrate that the performance of the proposed method comes at par with the performance of the traditional method by using lesser number of moment coefficients and thus results in significant saving in the feature extraction time.
\end{abstract}

\section{General Terms}

Feature extraction, Zernike moments, pseudo-Zernike moments, Face recognition.

\section{Keywords}

Zernike moments, pseudo-Zernike moments, Rotation invariance, Face recognition.

\section{INTRODUCTION}

Moments being powerful descriptors, help in capturing various image features. Feature extraction through moment function refers to extracting scalar values from the images. Some of the popularly used moment functions include Zernike Moments (ZMs), pseudo-Zernike moments (PZMs), orthogonal Fourier-Mellin moments (OFMMs) and radial harmonic Fourier moments (RHFMs). The selection of an appropriate feature extraction function is required in most of the applications of image processing and computer vision. The various application areas include image analysis, pattern recognition, watermarking, edge detection, face recognition, content based image retrieval, character recognition and so on. The application of these moments in the varied fields is based on their general properties like rotation invariance, scale invariance, robustness against noise, reconstruction capabilities etc. Invariance of the moment functions under geometrical transformations like rotation and scaling is most desirable in the recognition applications.

$\mathrm{Hu}$ [1] firstly introduced moment invariants using a linear combination of regular moments and were invariant under scaling, rotation and translation. Teague [2] defined Zernike moments based on the orthogonal Zernike polynomials. Orthogonality of ZMs attributes to the minimum information redundancy. Other important properties include robustness against transformational noise and excellent reconstruction capabilities. Owing to these properties, the ZMs were applied in the fields of character recognition [3], watermarking [4, 5], image retrieval [6], texture retrieval [7], face recognition [8] and image reconstruction [9]. Pseudo-Zernike moments (PZMs) were given by Bhatia and Wolf [10]. The behavior is similar to ZM but they are computationally more expensive as the number of coefficients for a given maximum order is double the number of coefficients of ZMs. The immunity to noise is better for PZMs. PZMs have been used in face recognition [11], optical character recognition [12],content based image retrieval [13], image watermarking [14], palm print verification [15], audio watermarking [16] etc. Sheng and Shen [17] introduced orthogonal Fourier-Mellin moments (OFMMs). Being orthogonal these moments have minimum overlapping of information. Also they are invariant under rotation and are resilient to image noise. They produce a larger number of coefficients as compared to other moments for the same maximum order, thus is more suitable in case of small images. The application areas include pattern recognition [18], character recognition [19], edge location [20], face recognition [21], image reconstruction [22] and so on. The RHFMs were introduced by Ren et al [23].The important properties possessed by RHFM are magnitude invariance, robustness to image noise, orthogonality and reconstruction capability. They are computationally less expensive and numerically more stable as compared to the other moment functions. Thus are applied in the fields such as watermarking [24, 25], color image retrieval [26], character reconstruction [27], tumor cell recognition [28] etc.

The magnitude invariance of the moments is crucial because the performance of the recognition applications depends on the behavior of the moments under deformations or transformations. The recognition is more accurate when the chosen feature extraction method is invariant to the transformations or deformations. The invariance property holds completely for the analog images but in the discrete domain because of the errors in computation, the property is not satisfied perfectly. Also the moments are computationally very expensive, thus feature extraction takes a considerable amount of time and the time taken is directly proportional to the number of coefficients being used for the application. In this paper we give an empirical analysis of the moment coefficients under rotation to find out the orders which are robust i.e. which follow the invariance property to a better extent. The more robust or stable moment coefficients are expected to increase the efficiency when used in the various recognition applications. The applicability of these moments in the varied fields and their importance in accurate recognition has motivated us to perform the empirical analysis on moment coefficients and results of the empirical analysis are applied to face recognition problem. 
The rest of the paper is structured as follows: Section 2 gives the mathematical description of the moment functions under consideration, Section 3 presents the traditional computational framework, Section 4 contains the empirical analysis of the moment coefficients under rotation, Section 5 gives the face recognition using empirical analysis and Section 6 provides the conclusion.

\section{MATHEMATICAL DESCRIPTION OF MOMENTS}

The moment coefficients are defined in the continuous polar domain over a unit disk. For an image function $f(x, y)$ of size $\mathrm{N} \times \mathrm{N}$ pixels the moment coefficient is given as:

$F_{p q}=\lambda \int_{0}^{2 \pi} \int_{0}^{1} f(r, \theta) V_{p q}^{*}(r, \theta) r d r d \theta$

where $0 \leq \mathrm{r} \leq 1$ and $0 \leq \Theta \leq 2 \pi$

' $p$ ' is the order, ' $q$ ' is the repetition, ' $\lambda$ ' is the normalization coefficient and $f(r, \Theta)$ is the pixel intensity. We can find $r=\sqrt{x_{f}^{2}+y_{f}^{2}}, \quad \Theta=\tan ^{-1}\left(\frac{y_{f}}{x_{f}}\right)$ from given $\left(x_{f}, y_{f}\right)$ in the Cartesian domain. The basis function $\mathrm{V}_{\mathrm{pq}}^{*}(\mathrm{r}, \theta)$ is the complex conjugate of basis function of particular type of moment which consists of two parts namely the angular part and the radial part.

$\mathrm{V}_{\mathrm{pq}}(\mathrm{x}, \mathrm{y})=\mathrm{V}_{\mathrm{pq}}(\mathrm{r}, \theta)=\mathrm{R}_{\mathrm{pq}}(r) * \mathrm{~A}_{\mathrm{q}}(\theta)$

Further

$\mathrm{A}_{\mathrm{q}}(\theta)=e^{i q \theta}=\cos (q \theta)+i \sin (q \theta)$

and $\mathrm{R}_{\mathrm{pq}}(r)$ which depends on ' $\mathrm{r}$ ', decides the type of moment.

\subsection{Zernike moments(ZMs)}

The Zernike moment $Z_{\mathrm{pq}}$ for a given image is defined in the continuous polar domain over a unit disk as:

$\mathrm{Z}_{\mathrm{pq}}=\frac{p+1}{\pi} \int_{0}^{2 \pi} \int_{0}^{1} \mathrm{f}(\mathrm{r}, \theta) \mathrm{V}_{\mathrm{pq}}^{*}(\mathrm{r}, \theta) \mathrm{rdr} \mathrm{d} \theta$

where the order ' $p$ ' and repetition ' $q$ ' are subjected to the following conditions:

$\mathrm{p} \geq 0,0 \leq|\mathrm{q}| \leq p, \mathrm{p}-|\mathrm{q}|=$ even and $\Theta=\tan ^{-1}\left(\frac{y}{x}\right)$

The radial part of the $\mathrm{R}_{\mathrm{pq}}(r)$ varies with the type of the moment function and for Zernike moment the radial polynomial function is given as:

$\mathrm{R}_{\mathrm{p} \mathrm{q}}^{Z M}(r)=\sum_{s=0}^{\frac{(p-|q|)}{2}} \frac{(-1)^{s}(p-s) !(r)^{p-2 s}}{s !\left(\frac{(p+|q|)}{2}-s\right) !\left(\frac{(p-|q|)}{2}-s\right) !}$

For a given maximum order $\mathrm{p}=p_{\max }$, the total number of $\mathrm{ZM}$ coefficients produced is:

$$
\left(1+p_{\max }\right)\left(2+p_{\max }\right) / 2
$$

\subsection{Pseudo-Zernike moments (PZMs)}

The basis function $V_{p q}(r, \Theta)$ of PZM is defined as:

$\mathrm{V}_{\mathrm{pq}}(\mathrm{r}, \theta)=\mathrm{R}_{\mathrm{p} \mathrm{q}}^{P Z M}(r) * e^{j q \theta}$

where the order ' $\mathrm{p}$ ' and repetition ' $\mathrm{q}$ ' are subjected to the conditions: $\mathrm{p} \geq 0,0 \leq|\mathrm{q}| \leq p$

The radial polynomial function PZMs differs from ZMs and is defined as:

$\mathrm{R}_{\mathrm{p} \mathrm{q}}^{P Z M}(r)=\sum_{s=0}^{\frac{(p-|q|)}{2}} \frac{(-1)^{s}(2 p+1-s) !(r)^{p-s}}{s !(p+|q|+1-s) !(p-|q|-s) !}$

For $p=p_{\max }$, the total number of PZM coefficients produced is $\left(1+p_{\max }\right)^{2}$

\subsection{Orthogonal Fourier-Mellin moments (OFMMs)}

The OFMM coefficient for a two dimensional image $f(r, \Theta)$ in the polar domain is computed as:

$\mathrm{O}_{\mathrm{pq}}=\frac{p+1}{\pi} \int_{0}^{2 \pi} \int_{0}^{1} \mathrm{f}(\mathrm{r}, \theta) \mathrm{V}_{\mathrm{pq}}^{*}(\mathrm{r}, \theta) \mathrm{r} \mathrm{dr} \mathrm{d} \theta$

Here order $p \geq 0$ and repetition $|q| \geq 0$. The radial function of OFMMs is expressed as:

$\mathrm{R}_{\mathrm{p} \mathrm{q}}^{O F M M}(r)=\sum_{s=0}^{p}(-1)^{p+s} \frac{(p+1+s) !(r)^{s}}{s !(p-s) !(s+1) !}$

For $p=p_{\text {max }}$, the number of OFMM coefficients is $(1+$ $\left.p_{\max }\right)\left(1+2 q_{\max }\right)$.

\subsection{Radial harmonic Fourier moments (RHFMs)}

The radial harmonic Fourier moments, $\mathrm{H}_{\mathrm{pq}}$, of order $\mathrm{p} \geq 0$ and repetition $|\mathrm{q}| \geq 0$ for a continuous image function, $\mathrm{f}(\mathrm{r}, \Theta)$, in the polar domain are defined over a unit disk as follows:

$\mathrm{H}_{\mathrm{p} \mathrm{q}}=\frac{1}{2 \pi} \int_{0}^{2 \pi} \int_{0}^{1} \mathrm{f}(\mathrm{r}, \Theta) \mathrm{V}_{\mathrm{pq}}^{*}(\mathrm{r}, \Theta) \mathrm{r} \mathrm{dr} \mathrm{d} \Theta$

The radial function of RHFMs is a sinusoidal function which is of the given form:

$$
\mathrm{R}_{\mathrm{p} \mathrm{q}}^{R H F M}(r)= \begin{cases}\frac{1}{\sqrt{r}} & p=0 \\ \sqrt{\frac{2}{r} \cos (\pi p r)} & p=\text { even } \\ \sqrt{\frac{2}{r} \sin (\pi(p+1) r)} & p=\text { odd }\end{cases}
$$

The number of RHFM coefficients generated for maximum order $p=p_{\max }$ is $\left(1+p_{\max }\right)\left(1+2 q_{\max }\right)$. 


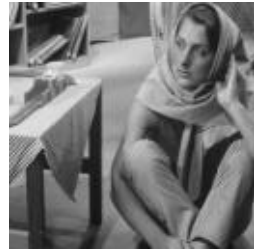

(a)

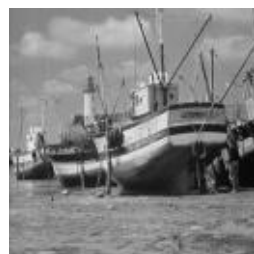

(e)

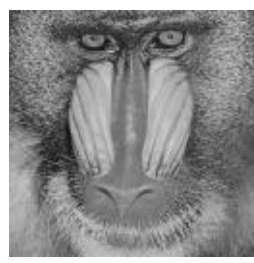

(i)

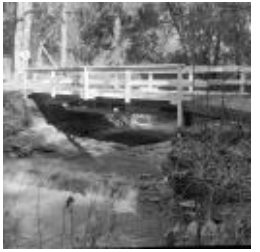

(b)

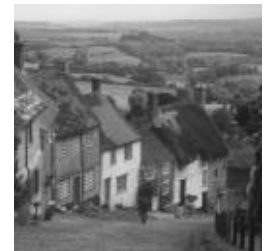

(f)

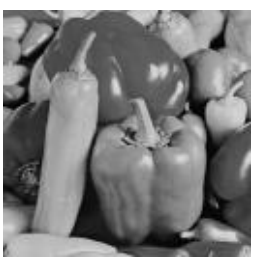

(j)

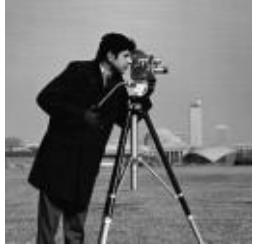

(c)

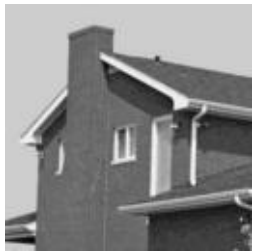

(g)

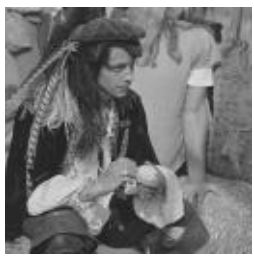

(k)

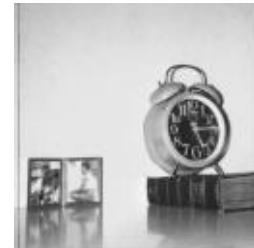

(d)

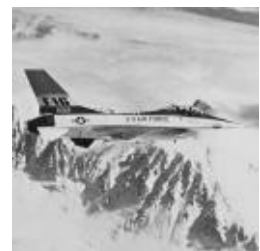

(h)

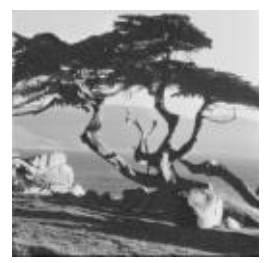

(1)

Figure 1: Twelve standard gray scale images used in the experiment (a) Barbara, (b) Bridge, (c) Cameraman, (d) Clock, (e) Fishing boat, (f) Gold hill, (g) House, (h) Jet plane, (i) Mandrill, (j) Peppers, (k) Pirate and (l) Tree.

\section{TRADITIONAL COMPUTATIONAL FRAMEWORK}

The discrete image $\mathrm{f}(\mathrm{x}, \mathrm{y})$ is usually given in Cartesian domain whereas the moment functions are defined in the continuous polar domain. Conversion of the image into polar domain is possible but it leads to violation of originality of the image and also introduces errors [29]. Thus the traditional method computes in the Cartesian domain using zeroth-order approximation given as:

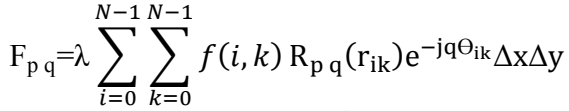

such that $\Delta \mathrm{x}=\Delta \mathrm{y}=\frac{2}{D}, \mathrm{r}_{\mathrm{ik}}^{2}=\mathrm{x}_{\mathrm{ik}}^{2}+\mathrm{y}_{\mathrm{ik}}^{2} \leq 1$ and $\Theta_{\mathrm{ik}}=$ $\tan ^{-1}\left(\frac{y_{k}}{x_{i}}\right)$

and the coordinates $\left(\mathrm{x}_{\mathrm{i}}, \mathrm{y}_{\mathrm{k}}\right)$ are defined as:

$x_{i}=\frac{2 i+1-N}{D} \quad y_{k=} \frac{2 k+1-N}{D}$

where $\mathrm{i}, \mathrm{k}=0,1, \ldots . \mathrm{N}-1$

Here $\mathrm{D}$ is the diameter of the circle and

$D=\left\{\begin{array}{l}N \quad \text { for inscribed circular disk } \\ N \sqrt{2} \quad \text { for outer circular disk }\end{array}\right.$

The mapping converts the square image into unit circular disk. The inscribed circular disk is contained in the square image and the outer circle contains the whole square image. The inscribed circle mapping has been used in our experiments.

\section{EMPIRICAL ANALYSIS OF MOMENT COEFFICIENTS UNDER ROTATION}

The discretization of moment function using zeroth-order approximation and the mapping of square image into unit disk introduces errors in the computation. These discretization errors affect the magnitude invariance property of the moments under the various transformations. The quantitative variation in the magnitude of the coefficients under rotation transformation can be computed as [30]:

$\sigma^{2}=\frac{1}{10} \sum_{i=1}^{10}\left(\frac{\left|F_{p q}\left(\theta_{i}\right)\right|}{\left|F_{p q}\left(\theta_{0}\right)\right|}-1\right)^{2}$

where $\theta_{i}=0^{\circ}, 5^{\circ}, 10^{\circ} \ldots ., 45^{\circ}$ and $F_{p q}\left(\theta_{i}\right)$ represents the value of moment at the angle $\theta_{i}$.

The quantitative variation, $\sigma^{2}$ is computed using Eq. (15) for the standard 256-level grayscale images of size $128 \times 128$ shown in Fig. 1. The maximum order $p_{\max }$ is assumed to be 10 for computing the various moment coefficients. Results from all the images are combined to find the Zernike moment coefficient which shows the highest quantitative variation and the least quantitative variation when subjected to rotation at multiple angles. Lesser quantitative variation indicates that the coefficient is more robust under rotation and vice versa.

The experiments were performed in the Microsoft's Visual Studio 2008 under Windows 7 environment on a PC with 2.66 $\mathrm{GHz}$ CPU and 4 GB RAM.

The average variation of each Zernike moment coefficient under rotation transformation is shown in Fig. 2. From Fig. 2 it is concluded that the variation is least for $\mathrm{Z}_{11}$ and highest 
for $Z_{91}$. The range of the quantitative variation gradually increases with the increase in moment order, that is, variation is greater for higher order moment coefficients. The average variation of each PZM, OFMM and RHFM coefficient is shown in Fig (3), (4) and (5) respectively.

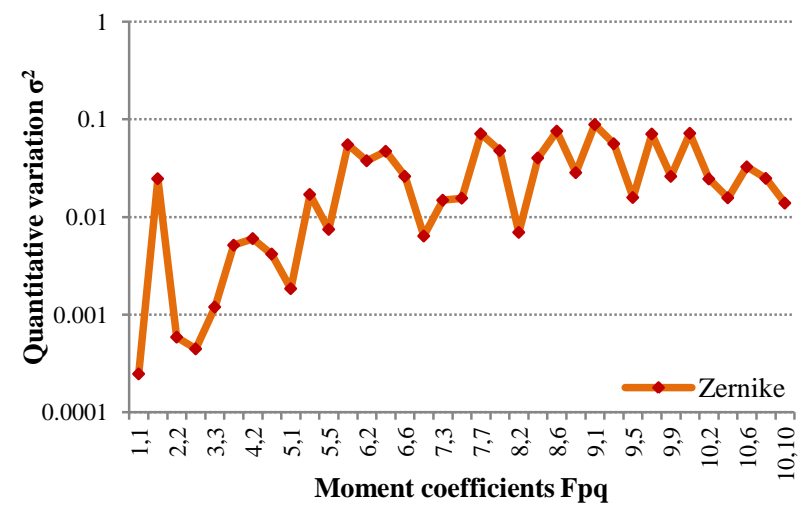

Figure 2: Quantitative variation of each ZM coefficient under rotation

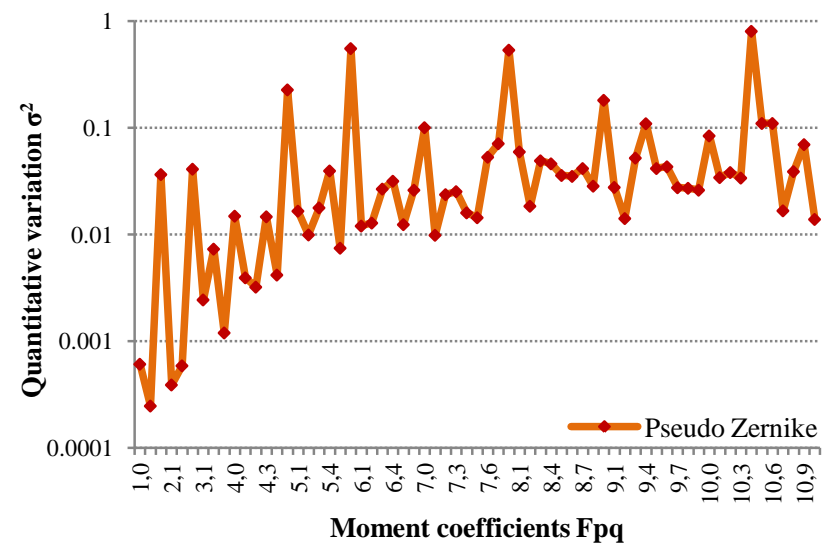

Figure 3: Quantitative variation of each PZM coefficient under rotation

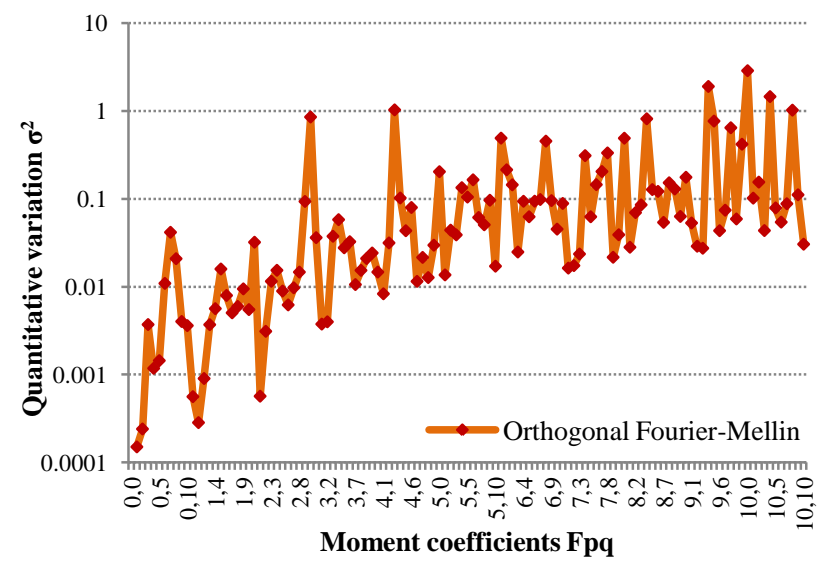

Figure 4: Quantitative variation of each OFMM coefficient under rotation

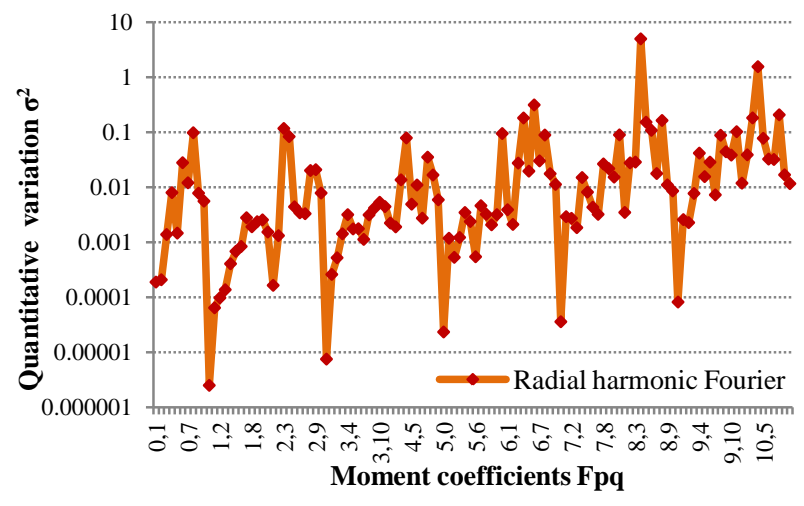

Figure 5: Quantitative variation of each RHFM coefficient under rotation

It is observed that PZMs (Fig.3) and OFMMs (Fig.4) also follow an upward trend for quantitative variation as order of the moment increases. The behavior of RHFMs (Fig.5) is different from the rest of the moment functions as the repetition ' 0 ' of the odd orders i.e. for $\mathrm{H}_{30}, \mathrm{H}_{50}, \mathrm{H}_{70}, \mathrm{H}_{90}$ shows least variations. The summary of the coefficients with least and highest variation for each of the moment function is given in Table1.

Table 1 Coefficients with least and highest variation under rotation

\begin{tabular}{ccc}
\hline $\begin{array}{c}\text { Moment } \\
\text { Function }\end{array}$ & $\begin{array}{c}\text { Coefficient with } \\
\text { least variation }\end{array}$ & $\begin{array}{c}\text { Coefficient with } \\
\text { highest variation }\end{array}$ \\
\hline ZM & $\mathrm{Z}_{11}$ & $\mathrm{Z}_{91}$ \\
PZM & $\mathrm{PZ}_{11}$ & $\mathrm{PZ}_{104}$ \\
OFMM & $\mathrm{O}_{01}$ & $\mathrm{O}_{100}$ \\
RHFM & $\mathrm{H}_{10}$ & $\mathrm{H}_{84}$ \\
\hline
\end{tabular}

It is also observed that some of the coefficients have exceptionally high quantitative variation for one or two images, while the variation is low for all the other images from Fig 1. For example $\mathrm{PZ}_{40}$ shows a high value of variation for Fig. $1(\mathrm{~g})$ and is low for all other images. Similarly, $\mathrm{Z}_{80}$ computes a high value of variation for Fig. 1(a) and is low for the rest of the images. Such observations suggest that the variation is partially dependent on the image under consideration.

Another parameter that has been used is $C_{p q}(\theta)$ which is a function of $\theta$ (the angle of rotation) and is computed as [30]:

$C_{p q}(\theta)=\frac{\left|F_{p q}(\theta)\right|}{\left|F_{p q}\left(0^{\circ}\right)\right|}$

where $\theta=0^{\circ}, 5^{\circ}, 10^{\circ} \ldots . .45^{\circ}$

The parameter $C_{p q}(\theta)$ is plotted against the angle of rotation for the coefficients with highest and least variation in order to compare the performances of the moments. 


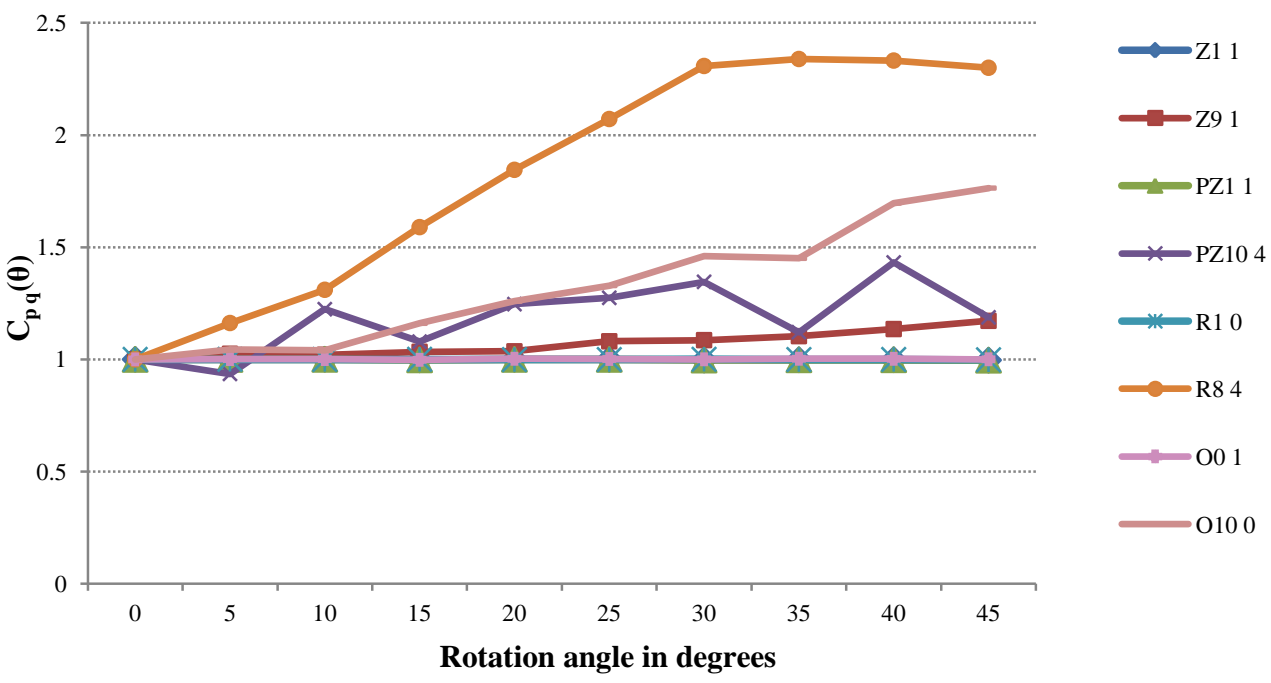

Figure 6: $C_{p q}(\theta)$ for the coefficients with highest and least variation.

From Fig.6 it is concluded that the ZM show lesser variation even in the worst case, i.e. $Z_{91}$ is more robust under rotation as compared to $\mathrm{PZ}_{10}, \mathrm{O}_{100}$ and $\mathrm{H}_{84}$. Thus it can be said that for any given set of images $\mathrm{ZM}$ provides best rotation invariance which is closely followed by PZM.

\section{FACE RECOGNITION USING EMPIRICAL ANALYSIS}

Face recognition is required in a wide variety of applications such as criminal identification, office security systems, video surveillance, authentication etc. The main challenges linked to face recognition are the variations in pose, facial expressions and illumination. It is important to select an efficient feature extraction method which is invariant to the changes or deformations in order to achieve a high accuracy face recognition system. The existing face recognition techniques are broadly classified as global feature extraction and local feature extraction methods. The global feature extraction methods give a holistic view of the image. Consequently they are more resistant to image noise, variations in pose, expressions etc. The local extraction methods on the other hand depict the local features and structures like eyes, mouth and nose and are more sensitive to noise. The moment based methods which fall under the category of global extraction methods fulfill the requirement of being invariant to the deformations or transformations like rotation. The moments that have earlier been used for this application are wavelet moments [31], Zernike moments [8, 32, 33] and pseudoZernike moments $[11,33]$. Out of these methods we have considered Zernike and pseudo-Zernike moments in our experiments.

It is a well known fact that the lower order coefficients give the crude aspects of the image while the higher order coefficients are responsible for extracting the fine details. For an efficient recognition a well balanced combination of both is required. It is clear from the empirical analysis that all the coefficients are not equally robust when subjected to rotation transformation. So a careful selection of a set of coefficients which are more robust under transformations may improve the performance of recognition system. It is required to find an optimum number of coefficients which provide a good tradeoff between the recognition rate and the time required for feature extraction [34]. Detailed experimentation has been performed on the ORL database and the description of the database is given below:

The Olivetti Research Laboratory (ORL) face database [35]The database has images of 40 subjects, each subject having 10 image variations thus a total of 400 images. The images are of size $92 \times 112$ pixels with 256 gray levels. The images of each subject vary in pose, illumination, expression, position and scale.

A subset of the ORL database has been used in our experiments. The images of first 20 subjects are considered. Half of the images i.e. the first 5 images of each subject are taken into the training set thus the training DB consists of 100 images. Remaining images have been manipulated as follows to form the test DB:

Rotation test DB- The last 5 images of each subject are resized to $128 \times 128$ pixels by adding canvas. Then the images are rotated at angles $10^{\circ}, 30^{\circ}, 50^{\circ}, 70^{\circ}$ and $90^{\circ}$. A sample image along with its rotated versions is given in Fig. 7. The rotated versions of the images are taken into rotation test set which makes the number of images in rotation test DB equal to 500 .
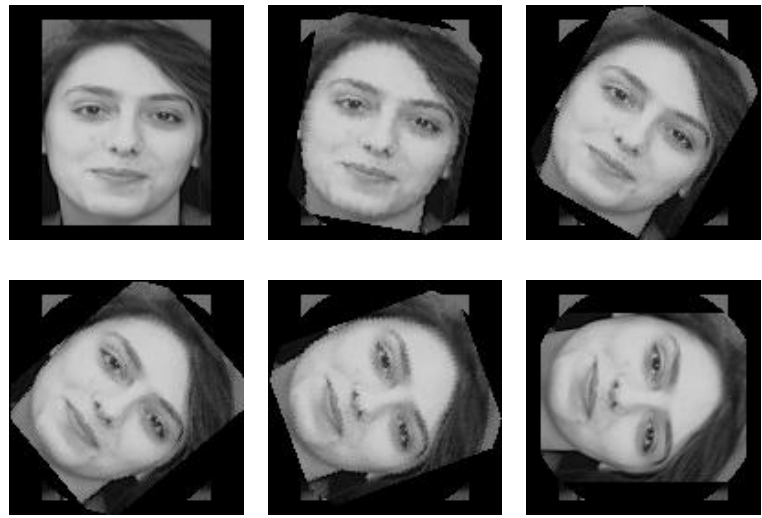

Figure 7: Sample image of subject from the ORL database and the rotated versions of the sample image. 
As mentioned in the previous section, it is observed that the variations computed for the individual coefficients under the transformations are partly dependent upon the images used. Thus for efficient face recognition the empirical analysis is performed on the training DB. The average variation of each coefficient of Zernike and pseudo-Zernike moments under rotation are computed using Eq. (15) for the 100 images in the training DB. The coefficients are then arranged according to increasing variations to form a sorted list of coefficients. The coefficients in the beginning of the list are more robust and the robustness decreases towards the end of the list. The recognition rate is computed using an increasing percentage of moment coefficients from the sorted list, starting with usage of 10 percent coefficients up to 100 percent. The aim is to find optimum number of coefficients required for achieving desired recognition rate. The distance metric used for comparing the extracted features is Euclidean distance or L2norm.

The sorted list of Zernike moment coefficients under rotation is given as follows:

$\left\{Z_{00}, Z_{22}, Z_{71}, Z_{31}, Z_{44}, Z_{62}, Z_{11}, Z_{91}, Z_{102}, Z_{66}, Z_{60}, Z_{51}\right.$,

$Z_{82}, Z_{33}, Z_{93}, Z_{88}, Z_{53}, Z_{99}, Z_{1010}, Z_{95}, Z_{104}, Z_{80}, Z_{55}, Z_{40}$,

$\left.\mathrm{Z}_{42}, \mathrm{Z}_{106}, \mathrm{Z}_{86}, \mathrm{Z}_{100}, \mathrm{Z}_{73}, \mathrm{Z}_{84}, \mathrm{Z}_{75}, \mathrm{Z}_{97}, \mathrm{Z}_{64}, \mathrm{Z}_{77}, \mathrm{Z}_{108}\right\}$

Similarly sorted list for pseudo-Zernike moment coefficients under rotation is given as follows:

$\left\{\mathrm{PZ}_{00}, \mathrm{PZ}_{10}, \mathrm{PZ}_{22}, \mathrm{PZ}_{20}, \mathrm{PZ}_{21}, \mathrm{PZ}_{51}, \mathrm{PZ}_{44}, \mathrm{PZ}_{11}, \mathrm{PZ}_{52}, \mathrm{PZ}_{41}\right.$,

$\mathrm{PZ}_{32}, \mathrm{PZ}_{42}, \mathrm{PZ}_{31}, \mathrm{PZ}_{66}, \mathrm{PZ}_{61}, \mathrm{PZ}_{62}, \mathrm{PZ}_{82}, \mathrm{PZ}_{33}, \mathrm{PZ}_{92}, \mathrm{PZ}_{71}$,

$\mathrm{PZ}_{81}, \mathrm{PZ}_{101}, \mathrm{PZ}_{88}, \mathrm{PZ}_{43}, \mathrm{PZ}_{74}, \mathrm{PZ}_{99}, \mathrm{PZ}_{1010}, \mathrm{PZ}_{61}, \mathrm{PZ}_{85}, \mathrm{PZ}_{75}$,

$\mathrm{PZ}_{105}, \mathrm{PZ}_{63}, \mathrm{PZ}_{86}, \mathrm{PZ}_{107}, \mathrm{PZ}_{93}, \mathrm{PZ}_{55}, \mathrm{PZ}_{97}, \mathrm{PZ}_{91}, \mathrm{PZ}_{60}, \mathrm{PZ}_{76}$,

$\mathrm{PZ}_{40}, \mathrm{PZ}_{72}, \mathrm{PZ}_{65}, \mathrm{PZ}_{84}, \mathrm{PZ}_{54}, \mathrm{PZ}_{103}, \mathrm{PZ}_{90}, \mathrm{PZ}_{95}, \mathrm{PZ}_{104}, \mathrm{PZ}_{106}$,

$\mathrm{PZ}_{73}, \mathrm{PZ}_{94}, \mathrm{PZ}_{80}, \mathrm{PZ}_{87}, \mathrm{PZ}_{64}, \mathrm{PZ}_{108}, \mathrm{PZ}_{98}, \mathrm{PZ}_{100}, \mathrm{PZ}_{50}, \mathrm{PZ}_{77}$

$\left.\mathrm{PZ}_{109}, \mathrm{PZ}_{96}, \mathrm{PZ}_{30}, \mathrm{PZ}_{70}\right\}$

The recognition performance of Zernike moments on the rotation test DB is shown in Fig. 8. The recognition rate increases sharply in the beginning as the percentage of coefficients used increases but after the usage of 60 percent of the coefficients the increase in the recognition rate is marginal that is, the inclusion of more coefficients does not make any significant change in the recognition rate. The recognition performance of pseudo-Zernike coefficients is shown in Fig. 9. A similar pattern is observed where the recognition rate increases initially but after the inclusion of 60 percent of coefficients the recognition rate reaches saturation. The time required for feature extraction at different stages for Zernike and pseudo-Zernike moments are given in Table 2 and Table 3 respectively.

From the Fig. 8 and Fig. 9 we can conclude that around 60 percent of coefficients of Zernike and pseudo-Zernike moment coefficients are sufficient for efficient face recognition under in-plane rotation. The percentage saving in time in this scenario is quiet considerable and will be even more significant when the size of the training and test DB is larger. The percentage saving in feature extraction time is approximately $25 \%$ in case of Zernike moment coefficients and for pseudo-Zernike moments it is around $31 \%$. The comparison of the performance of the proposed and traditional method is given in Table 4 .

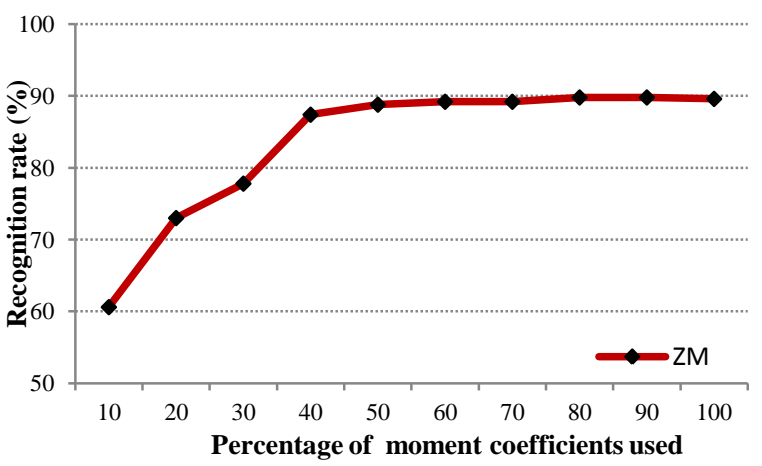

Figure 8: Recognition rate versus percentage of Zernike coefficients used for rotation DB

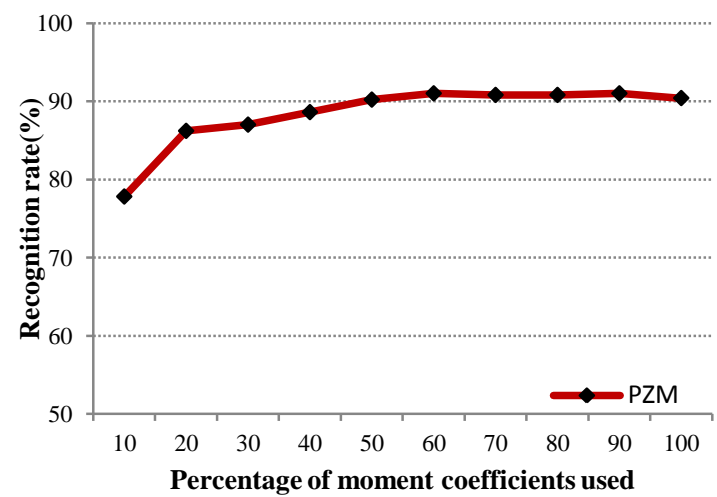

Figure 9: Recognition rate versus percentage of pseudoZernike coefficients used for rotation DB

Table 2 Recognition rate for Zernike moment for rotation test DB

\begin{tabular}{cccc}
\hline $\begin{array}{l}\text { Percentage } \\
\text { of } \\
\text { coefficients } \\
\text { used }\end{array}$ & $\begin{array}{l}\text { Recognition } \\
\text { rate }(\%)\end{array}$ & $\begin{array}{l}\text { Feature } \\
\text { extraction } \\
\text { time(sec)for } \\
\text { training DB }\end{array}$ & $\begin{array}{l}\text { Feature } \\
\text { extraction } \\
\text { time(sec)for } \\
\text { test DB }\end{array}$ \\
\hline 10 & 60.6 & 2.73 & 13.26 \\
20 & 73 & 3.13 & 15.15 \\
30 & 77.8 & 3.43 & 16.93 \\
40 & 87.4 & 3.88 & 18.99 \\
50 & 88.8 & 4.23 & 20.92 \\
60 & 89.2 & 4.76 & 23.49 \\
70 & 89.2 & 5.07 & 24.85 \\
80 & 89.8 & 5.49 & 27.30 \\
90 & 89.8 & 5.80 & 28.97 \\
100 & 89.6 & 6.29 & 31.39 \\
\hline
\end{tabular}


Table 3 Recognition rate for pseudo-Zernike moment for rotation test DB

\begin{tabular}{cccc}
\hline $\begin{array}{l}\text { Percentage } \\
\text { of } \\
\text { coefficients } \\
\text { used }\end{array}$ & $\begin{array}{l}\text { Recognition } \\
\text { rate }(\%)\end{array}$ & $\begin{array}{l}\text { Feature } \\
\text { extraction } \\
\text { time(sec)for } \\
\text { training DB }\end{array}$ & $\begin{array}{l}\text { Feature } \\
\text { extraction } \\
\text { time(sec)for } \\
\text { test DB }\end{array}$ \\
\hline 10 & 77.8 & 3.03 & 14.52 \\
20 & 86.2 & 3.59 & 17.38 \\
30 & 87 & 4.26 & 20.98 \\
40 & 88.6 & 4.84 & 24.27 \\
50 & 90.2 & 5.72 & 28.13 \\
60 & 91 & 6.32 & 31.54 \\
70 & 90.8 & 6.86 & 34.54 \\
80 & 90.8 & 7.65 & 38.56 \\
90 & 91 & 8.35 & 42.09 \\
100 & 90.4 & 9.05 & 45.89 \\
\hline
\end{tabular}

A slight drop in recognition rate is observed towards the end in Fig.8 and Fig.9. This can be termed as an anomalous behavior as it is expected that the recognition rate should increase with the increase in percentage of coefficients used for recognition. This behavior is due to the inclusion of more sensitive moment coefficients which are unstable under rotation transformation. The anomalous behavior is also attributed to the inaccuracy in computation of moment coefficients arising due to the geometrical error and numerical instability when computed using zeroth-order approximation. In future the use of more accurate and numerically stable methods for computation of moments can be used to overcome the anomalous behavior.

Table 4 Comparison of performances of Proposed and Traditional Method

\begin{tabular}{|c|c|c|c|c|}
\hline Moment & $\begin{array}{l}\text { Comparison } \\
\text { parameter }\end{array}$ & $\begin{array}{l}\text { Proposed } \\
\text { method }\end{array}$ & $\begin{array}{l}\text { Traditional } \\
\text { method }\end{array}$ & $\begin{array}{l}\text { Saving } \\
\text { in time } \\
(\%)\end{array}$ \\
\hline \multirow{2}{*}{$\mathbf{Z M}$} & $\begin{array}{c}\text { Feature } \\
\text { extraction } \\
\text { time }(\mathrm{sec})\end{array}$ & 28.25 & 37.68 & \multirow{2}{*}{25} \\
\hline & $\begin{array}{l}\text { Recognition } \\
\text { rate }(\%)\end{array}$ & 89.2 & 89.6 & \\
\hline \multirow{2}{*}{ PZM } & $\begin{array}{c}\text { Feature } \\
\text { extraction } \\
\text { time(sec) }\end{array}$ & 37.86 & 54.94 & \multirow{2}{*}{31} \\
\hline & $\begin{array}{l}\text { Recognition } \\
\text { rate }(\%)\end{array}$ & 91 & 90.4 & \\
\hline
\end{tabular}

\section{CONCLUSION}

Moment functions have been extensively used for feature extraction from images. Owing to the magnitude invariance of these functions under geometrical transformations, they have been used in recognition problems. In this paper a comparison of various moment functions under rotation is given using an empirical analysis of each moment function. ZM and PZM were found to give best rotation invariance and were used for face recognition under in-plane rotation. Using the empirical analysis an optimum number of moment coefficients were selected. The recognition rate comes at par with that of the traditional method using lesser number of coefficients. As the moment coefficients are computationally expensive, the reduced number of coefficients results in a percentage saving in time of around $25 \%$ for ZMs and $31 \%$ for PZMs which is even more significant in the case of large databases.

\section{REFERENCES}

[1] M.K. Hu, "Visual Pattern Recognition by Moment Invariants," IRE transaction on Information Theory, vol. 8, pp. 179-187, 1962.

[2] M. R. Teague, "Image analysis via general theory of moments," Journal of Optical Society of America, vol. 70, no. 8, pp. 920-30, 1980.

[3] A. Broumandnia and J. Shanbehzadeh, "Fast Zernike wavelet moments for Farsi character recognition," Image and Vision Computing, vol. 25, pp. 717-726, 2007.

[4] H. S. Kim and H.K. Lee, "Invariant Image Watermark Using Zernike Moments," IEEE Transactions on Circuits and Systems for Video Technology, vol. 13, no. 8, pp. 766-775, 2003.

[5] N. Singhal, Y.Y. Lee, C.S. Kim and S.U. Lee, "Robust image watermarking using local Zernike moments," $J$. Vis. Commun. Image R., vol. 20, pp. 408-419, 2009.

[6] C. Singh and Pooja, "Improving image retrieval using combined features of Hough transform and Zernike moments," Optics and Lasers in Engineering, vol. 49, pp. 1384-1396, 2011.

[7] D.G. Sima, H.K. Kimb and R.H. Park, "Invariant texture retrieval using modified Zernike moments," Image and Vision Computing, vol. 22, pp. 331-342, 2004.

[8] S. Farokhia, S. M. Shamsuddin, U. Sheikhb, J. Flusser, M. Khansarid and K. Jafari-Khouzani, "Near infrared face recognition by combining Zernike moments and undecimated discrete wavelet transform," Digital Signal Processing, vol. 31, pp. 13-27, 2014.

[9] S. X. Liao, "Image Analysis by Moments,Ph.D. Thesis," University of Manitoba, Winnipeg, Manitoba,Canada, 1993.

[10] A. Bhatia and E. Wolf, " On the circle polynomials of Zernike and related orthogonal sets," Mathematical Proceedings of the Cambridge Philosophical Society, vol. 50, pp. 40-48, 1954.

[11] Y.H. Pang, A. B. Teoh and D. C. Ngo, "A Discriminant Pseudo Zernike Moments in Face Recognition," Journal of Research and Practice in Information Technology, vol. 38, no. 2, pp. 197-211, 2006.

[12] R. Bailey and M. Srinath, "Orthogonal moment features for use with parametric and non-parametric classifiers," IEEETrans.PatternAnal.Mach.Intell., vol. 18, no. 4, p. 389-399, 1996.

[13] X.Y. Wang, Y.J. Yu and H.Y. Yang, "An effective image retrieval scheme using color,texture and shape features," Comput.Stand.Interfaces, vol. 33, no. 1, p. 59-68, 2011.

[14] Y. Xin, S. Liao and M. Pawlak, "Geometrically robust image watermark via pseudo-Zernike moments," in IEEE Canadian Conference on Electrical and Computer Engineering (CCECE), Canada, 2004.

[15] Y. Pang, T. Andrew, N. David and F. Hiew, "Palmprint verifications with moments,", Journal ofWSCG, vol. 12, pp. 1-3, 2003. 
[16] X. Wang, T. Ma and P. Niu, "A pseudo-Zernike moment based audio watermarking scheme robust against desynchronization attacks," Comput.Electr.Eng., vol. 37, p. 425-443, 2011.

[17] Y. Sheng and L. Shen, "Orthogonal Fourier-Mellin moments for invariant pattern recognition," Journal of Optical Society of America., vol. 11, no. 6, pp. 17481757, 1994.

[18] H. Zhang, H. Shu, P. Haigron, B. Li and L. Luo, "Construction of a complete set of orthogonal FourierMellin moment invariants for pattern recognition applications," Image and Vision Computing, vol. 28 , p. $38-44,2010$

[19] C. Kan and M. D. Srinath, "Invariant character recognition with Zernike and orthogonal Fourier-Mellin moments," Pattern Recognition, vol. 35, pp. 143-154, 2002.

[20] T. Bin, A. Lei, C. Jiwen, K. Wenjing and L. Dandan, "Subpixel edge location based on orthogonal FourierMellin moments," Image and Vision Computing, vol. 26, pp. $563-569,2008$.

[21] Y. M. Chen and J.-H. Chiang, "Face recognition using combined multiple feature extraction based on FourierMellin approach for single example image per person," Pattern Recognition Letters, vol. 31, p. 1833-1841, 2010.

[22] G. Papakostas, B. Mertzios and D. Karras, "Performance of the orthogonal moments in reconstructing biomedical images," in Systems,signals and image processing IWSSIP. 16th conference on, Chalkida, Greece, 2009.

[23] H. Ren, Z. Ping, W. Bo, W. Wu and Y. Sheng, "Multidistortion-invariant image recognition with radial harmonic Fourier moments.," Journal of Optical Society of America, vol. 20, no. 4, pp. 631-637, 2003.

[24] C. Singh and S. K. Ranade, "A high capacity image adaptive watermarking scheme with radial harmonicFourier moments," Digital Signal Processing, vol. 23, p. 1470-1482, 2013.

[25] Y. Hong-Yinga, W. Xiang-Yanga, W. Peia and PanPana, "Geometrically resilient digital watermarking scheme based on radialharmonic Fourier moments magnitude," International Journal of Electronics and Communications, vol. 69, p. 389-399, 2015.
[26] W. Xiang-yang, W.Y. Li, H.Y. ,. N. Pan-pan and L. Yong-wei, "Invariant quaternion radial harmonic Fourier moments for color image retrieval," Optics \& Laser Technology, vol. 66, p. 78-88, 2015.

[27] H. Ren, A. Liu, J. Zou, D. Bai and Z. Ping, "Character reconstruction with Radial harmonic Fourier moments," in Fourth International Conference on Fuzzy Systems and Knowledge Discovery (FSKD 2007), China, 2007.

[28] H. Ren, A. Liu, J. Zou, Z. Ping and D. Bai, "Study on a novel tumor cell recognition system based on orthogonal image moments," in 7th Asian-Pacific Conference on Medical and Biological Engineering, Beijing, China, 2008.

[29] C. Singh and E. Walia, "Computation of Zernike moments in improved Polar Configuration," Image processing ,IET, vol. 3, no. 4, pp. 217-227, 2009.

[30] C. Singh, S. Pooja and R. Upneja, "On Image Reconstruction, Numerical Stability, and Invariance of Orthogonal Radial Moments and Radial Harmonic Transforms," Pattern Recognition and Image Analysis, vol. 21, no. 4, pp. 663-676, 2011.

[31] C. Singh and A. M. Sahan, "Face recognition using complex wavelet moments," Optics \& Laser Technology, vol. 47, pp. 256-267, 2013.

[32] N. H. Foon, Y.H. Pang, A. T. B. Jin and D. N. C. Ling, "An efficient method for human face recognition using wavelet transform and Zernike moments.," in Computer Graphics, Imaging and Visualization, CGIV 2004. Proceedings. International Conference on, Washington,U.S.A, 2004.

[33] C. Singh, E. Walia and N. Mittal, "Discriminative Zernike and Pseudo Zernike Moments for Face Recognition," International Journal of Computer Vision and Image Processing (IJCVIP), vol. 2, no. 2, pp. 12-35, 2012.

[34] C. Singh and A. Aggarwal, "A noise resistant image matching method using angular radial transform," Digital Signal Processing, vol. 33, p. 116-124, 2014.

[35] "The Database of Faces," AT\&T Laboratories, 1994. [Online]. Available: http://www.cl.cam.ac.uk/research/dtg/attarchive/facedat abase.html. [Accessed 14 March 2015]. 